\title{
Combination of the Improved Diffraction Nonlocal Boundary Condition and Three-Dimensional Wide-Angle Parabolic Equation Decomposition Model for Predicting Radio Wave Propagation
}

\author{
Ruidong Wang, Guizhen Lu, Rongshu Zhang, and Weizhang Xu \\ Communication University of China, Beijing 100024, China \\ Correspondence should be addressed to Ruidong Wang; 123495518@qq.com
}

Received 14 April 2017; Revised 23 July 2017; Accepted 20 August 2017; Published 28 September 2017

Academic Editor: Sotirios K. Goudos

Copyright (C) 2017 Ruidong Wang et al. This is an open access article distributed under the Creative Commons Attribution License, which permits unrestricted use, distribution, and reproduction in any medium, provided the original work is properly cited.

Diffraction nonlocal boundary condition (BC) is one kind of the transparent boundary condition which is used in the finitedifference (FD) parabolic equation (PE). The greatest advantage of the diffraction nonlocal boundary condition is that it can absorb the wave completely by using one layer of grid. However, the speed of computation is low because of the time-consuming spatial convolution integrals. To solve this problem, we introduce the recursive convolution (RC) with vector fitting (VF) method to accelerate the computational speed. Through combining the diffraction nonlocal boundary with RC, we achieve the improved diffraction nonlocal BC. Then we propose a wide-angle three-dimensional parabolic equation (WA-3DPE) decomposition algorithm in which the improved diffraction nonlocal BC is applied and we utilize it to predict the wave propagation problems in the complex environment. Numeric computation and measurement results demonstrate the computational accuracy and speed of the WA-3DPE decomposition model with the improved diffraction nonlocal BC.

\section{Introduction}

Electromagnetic lateral propagation, which includes lateral scattering, lateral diffraction, and the depolarization effect, affects the actual propagation in irregular terrains, especially in situations with steep transverse gradients. In this situation, the two-dimensional (2D) parabolic equation (PE) is no longer accurate. In order to get more accurate results, the three-dimensional parabolic equation (3DPE) is adopted. The 3DPE has two forms of scalar and vector, which comes from the scalar and vector wave equation, respectively. The scalar 3DPE has only one equation that can just reflect the propagation characteristics of an electromagnetic field in a particular component in $3 \mathrm{D}$ space. By contrast, vector 3DPE is composed of three equations, which can include all field components, with their boundary conditions. Levy [1] first used scalar 3DPE to calculate the diffraction problem behind urban buildings. Saini and Casiragh [2] studied the 3D split-step Fourier transformation method, in which a 2D plane Fourier transformation was used, and the plane was perpendicular to the propagation direction. Compared with the 3D finite-difference method, 3D split-step Fourier transformation method decreased computational complexity, but the computational amount was still very large for largescale computing problems. Zelley and Constantinou [3] established a 3DPE model based on implicit finite-difference method. The implicit finite-difference method can deal with the boundary conditions flexibly; therefore, it is relatively simple to solve the radio wave propagation problem on different terrains. However, due to the large-scale matrix operation, the computation amounts are still very large and the demand for resource is high. In recent years, using 3DPE to predict the radio wave propagation in a small area such as in the urban district environment has become a hot topic and has achieved many results [4-6].

The gradual improvement of the 3D geographic information system in recent years has caused the large-scale 3D electromagnetic propagation prediction to become a trend. 
Similar to the case of the 2DPE development, the computational amount of the 3DPE is the main obstacle, especially for radio wave propagation on irregular terrains. The convergence rate depends on the height and lateral width of the computational domain. For large-scale and real-time computation, there still exists a considerable challenge. To accelerate the computational speed of the scalar wide-angle 3DPE (WA3DPE), we propose a decomposition algorithm. According to this algorithm, the total field at the receiving point is approximately the sum of the straight wave and diffraction wave with the shortest propagation path. In our proposed algorithm, the WA-3DPE can be decomposed into two wideangle 2DPEs which is called a WA-3DPE decomposition model. The WA-3DPE decomposition model can deal with the horizontal diffraction and vertical diffraction caused by irregular terrain obstructions simultaneously. Furthermore this approximation can largely decrease the computational complexity of the WA-3DPE and can accelerate the speed of computation.

Boundary condition (BC) is critical to the use of PE for accurate prediction of radio wave propagation. The $2 \mathrm{DPE}$ prediction of radio wave propagation involves two BCs, which are the upper $\mathrm{BC}$ and the lower BC, respectively. For the lower BC, before the 1990s, the smooth and perfect conducting $\mathrm{BC}$ was adopted. However, the ideal conducting BC caused large errors in practice. Until 1991, the introduction of hybrid Fourier transform method [7] made it possible to apply impedance $\mathrm{BC}$ in the solution PE. For non-PEC surfaces and buildings, the fields on them generally meet the impedance BC. For the upper boundary condition, the most used BCs include window function BC [8], perfect matching layers [9], and transparent $\mathrm{BC}[10,11]$. For the 3DPE, it contains four BCs. It needs to consider the left and right BCs as well as the upper and lower BCs like the 2DPE. And the most used left and right BCs of 3DPE are the window function BCs [12].

The nonlocal BC is an exact boundary that does not require the additional computational space in the form of absorbing layers. In the previous studies [13], we used the traditional nonlocal boundary conditions in wide-angle two-dimensional parabolic equation model to calculate the propagation loss. However, it has the disadvantage that the 2DPE ignored the lateral scattering and diffraction, and, due to the presence of a spatial convolution integral, the computation is time-consuming. This computation is also memory demanding as it requires the storage and use of all previous values of the field along the boundary. To solve this problem, [14] proposed the recursive convolution (RC) formulation to reduce the computational burden. The $\mathrm{RC}$ was achieved using the vector fitting (VF) method. The nonlocal BC combined with RC formulation was applied in a wide-angle 2DPE based on the FD solving method. The computational speed and the accuracy of the nonlocal $\mathrm{BC}$ combined with $\mathrm{RC}$ formulation were demonstrated. For the actual radio wave propagation problem, the initial source is often fixed at the start position, and there is no need to use the nonlocal BC. Using the simpler diffractive nonlocal BC [15] is enough. Therefore, we adopt the $\mathrm{RC}$ formulation to improve the computational speed of the diffraction nonlocal BC. As the diffraction nonlocal BC is a simplified form of the nonlocal BC, the $\mathrm{RC}$ formulation can be applied directly from nonlocal BC to diffraction nonlocal BC. The RC formulation is applicable to the nonlocal $\mathrm{BC}$ as well as to the corresponding simpler diffractive nonlocal BC. We call the diffraction nonlocal BC combined with RC as improved diffraction nonlocal BC. We will apply this improved diffraction nonlocal BC in the WA3DPE decomposition model (described in Section 2).

\section{WA-3DPE Decomposition Model}

In 3D Cartesian coordinate system, if radio wave propagates along the positive direction of $x$-axis, the 3DPE [15] can be written as

$$
\frac{\partial u(x, y, z)}{\partial x}=-i k_{0}(1-\sqrt{1+q}) u(x, y, z),
$$

where $q=1 / k_{0}^{2}\left(\partial^{2} / \partial_{y}^{2}+\partial^{2} / \partial_{z}^{2}\right)+n^{2}-1,\left(k_{0}=2 \pi / \lambda\right)$ is the wave number in the free space, and $n$ is the refractive index. Equation (1) corresponds to forward propagating wave.

For numerical implementation, we need a suitable approximation of the $q$ operator. With the help of Padé approximation [15],

$$
\sqrt{1+q} \approx \frac{(1+0.75 q)}{(1+0.25 q)}
$$

a WA-3DPE is obtained.

$$
\begin{aligned}
& \left\{\left(1+\frac{1}{4}\left(n^{2}-1\right)\right) \frac{\partial}{\partial x}+\frac{1}{4 k_{0}^{2}} \frac{\partial}{\partial x}\left(\frac{\partial^{2}}{\partial y^{2}}+\frac{\partial^{2}}{\partial z^{2}}\right)\right. \\
& \left.+\frac{i}{2 k_{0}}\left(\frac{\partial^{2}}{\partial y^{2}}+\frac{\partial^{2}}{\partial z^{2}}\right)+\frac{i}{2}\left(n^{2}-1\right)\right\} u(x, y, z)=0 .
\end{aligned}
$$

Equation (3) is called the Claerbout WA-3DPE, which keeps the propagation angle up to 40 degrees. Although the WA$3 \mathrm{DPE}$ can model radio wave propagation in $3 \mathrm{D}$ space, the computing efficiency is low because of large-scale matrix operations.

In order to accelerate the WA-3DPE speed of computation, similar to the ray tracing method, the main consideration is given to the straight wave and diffraction wave with the shortest propagation path, which can largely decrease the computational complexity of the WA-3DPE. It is assumed that the $3 \mathrm{D}$ function $u(x, y, z)$ can be expressed as the sum of two 2D functions:

$$
u(x, y, z) \approx u_{h}(x, y)+u_{v}(x, z),
$$

where $u_{h}(x, y)$ is propagation waves in $(x, y)$ plane and $u_{v}(x, z)$ is propagation waves in $(x, z)$ plane. By applying $(4)$, (3) can be reduced to two 2DPEs:

$$
\begin{aligned}
& \left\{\left(1+\frac{1}{4}\left(n^{2}-1\right)\right) \frac{\partial}{\partial x}+\frac{1}{4 k_{0}^{2}} \frac{\partial^{3}}{\partial x \partial y^{2}}+\frac{i}{2 k_{0}} \frac{\partial^{2}}{\partial y^{2}}\right. \\
& \left.+\frac{i k_{0}}{2}\left(n^{2}-1\right)\right\} u_{h}(x, y)=0,
\end{aligned}
$$




$$
\begin{aligned}
& \left\{\left(1+\frac{1}{4}\left(n^{2}-1\right)\right) \frac{\partial}{\partial x}+\frac{1}{4 k_{0}^{2}} \frac{\partial^{3}}{\partial x \partial z^{2}}+\frac{i}{2 k_{0}} \frac{\partial^{2}}{\partial z^{2}}\right. \\
& \left.+\frac{i k_{0}}{2}\left(n^{2}-1\right)\right\} u_{v}(x, z)=0 .
\end{aligned}
$$

Equations (5) and (6) are called WA-3DPE decomposition model. The WA-3DPE decomposition model actually contains two wide-angle 2DPEs. The total field at the receiving point is the sum of $u_{h}(x, y)$ and $u_{v}(x, z)$.

\section{Finite-Difference Method of the WA-3DPE Decomposition Model}

Since (5) and (6) are symmetrical in form, here we only take (5) as an example to introduce finite-difference implementation, and the finite-difference implementation of (6) can be obtained in the same way. We start by defining the integration grid, which is fixed in the $y$ direction, but not in the $x$ direction, so that it can adapt to the terrain shape. We let $y_{n h}=n h \cdot \Delta y, n h=0, \ldots, N h$ be the grid point at $y$ direction and $x_{m}=m \cdot \Delta x, m=0, \ldots, M, \ldots$ be the successive integration ranges. Using the central difference approximation, the derivatives of (5) are discretized as follows:

$$
\begin{gathered}
\varepsilon_{m}=\frac{x_{m-1}+x_{m}}{2}, \\
u_{h}\left(\varepsilon_{m}, y_{n h}\right)=\frac{u_{h}\left(x_{m}, y_{n h}\right)+u_{h}\left(x_{m-1}, y_{n h}\right)}{2} .
\end{gathered}
$$

In order to advance the solution from range $x_{m-1}$ to range $x_{m}$, (7) and (8) give the midpoint

$$
\frac{\partial u_{h}}{\partial x}\left(\varepsilon_{m}, y_{n h}\right)=\frac{u_{h}\left(x_{m}, y_{n h}\right)-u_{h}\left(x_{m-1}, y_{n h}\right)}{\Delta x}
$$

Equation (9) gives the central finite-difference approximation of the derivative in range

$$
\begin{aligned}
& \frac{\partial^{2} u_{h}}{\partial y^{2}}\left(\varepsilon_{m}, y_{n h}\right) \\
& \quad=\frac{u_{h}\left(\varepsilon_{m}, y_{n h+1}\right)+u_{h}\left(\varepsilon_{m}, y_{n h-1}\right)-2 u_{h}\left(\varepsilon_{m}, y_{n h}\right)}{\Delta y^{2}} .
\end{aligned}
$$

Equation (10) gives the central finite-difference approximation of the second-order derivative in $y$ :

$$
\begin{aligned}
& \frac{\partial^{3} u_{h}}{\partial x \partial y^{2}}\left(x_{m}, y_{n h}\right) \\
& \quad=\frac{u_{h}\left(x_{m}, y_{n h+1}\right)+u_{h}\left(x_{m}, y_{n h-1}\right)-2 u_{h}\left(x_{m}, y_{n h}\right)-u_{h}\left(x_{m-1}, y_{n h+1}\right)-u_{h}\left(x_{m-1}, y_{n h-1}\right)+2 u_{h}\left(x_{m-1}, y_{n h}\right)}{\Delta x \Delta y^{2}} .
\end{aligned}
$$

Equation (11) gives the central finite-difference approximation of the third-order mixed derivative.

Substituting (8)-(11) in (5) yields

$$
\begin{aligned}
& \alpha_{h, m} u_{h, n h+1}^{m}+\beta_{h, m} u_{h, n h}^{m}+\alpha_{h, m} u_{h, n h-1}^{m} \\
& =\alpha_{h, m-1} u_{h, n h+1}^{m-1}-\beta_{h, m-1} u_{h, n h}^{m-1}-\alpha_{h, m-1} u_{h, n h-1}^{m-1},
\end{aligned}
$$

where we have used the following notations:

$$
\begin{aligned}
\alpha_{h, m}= & \frac{1+j k_{0} \Delta x}{4 k_{0}^{2}(\Delta y)^{2}}, \\
\alpha_{h, m-1}= & \frac{1-j k_{0} \Delta x}{4 k_{0}^{2}(\Delta y)^{2}}, \\
\beta_{h, m}= & 1+\frac{1}{4}\left(n^{2}\left(\varepsilon_{m}, y_{n h}\right)-1\right)\left(1+j k_{0} \Delta x\right) \\
& -\frac{1+j k_{0} \Delta x}{2 k_{0}^{2}(\Delta y)^{2}}, \\
\beta_{h, m-1}= & 1+\frac{1}{4}\left(n^{2}\left(\varepsilon_{m}, y_{n h}\right)-1\right)\left(1+j k_{0} \Delta x\right) \\
& -\frac{1-j k_{0} \Delta x}{2 k_{0}^{2}(\Delta y)^{2}} .
\end{aligned}
$$

Equation (12) has expressed values at range $x_{m}$ as a function of values at range $x_{m-1}$ in the form of a linear system. When $m=1$, (12) can be written as

$$
\begin{aligned}
& \alpha_{h, 1} u_{h, n h+1}^{1}+\beta_{h, 1} u_{h, n h}^{1}+\alpha_{h, 1} u_{h, n h-1}^{1} \\
& =\alpha_{h, 0} u_{h, n h+1}^{0}-\beta_{h, 0} u_{h, n h}^{0}-\alpha_{h, 0} u_{h, n h-1}^{0} .
\end{aligned}
$$

For the Gaussian source,

$$
\begin{gathered}
u_{h, n h}^{0}=u_{h}(0, n h \cdot \Delta z)=u_{h}(0, z)=A \frac{k_{0} B}{2 \sqrt{2 \pi \log 2}} \\
\cdot \exp \left(-j k_{0} \theta z\right) \exp \left(-\frac{B^{2}}{8 \log 2} k_{0}^{2}\left(z-z_{s}\right)^{2}\right),
\end{gathered}
$$

where $A$ is a normalization constant; $B$ is half-power beamwidth; $\theta$ is elevation angle; and $z_{s}$ is transmitting antenna height.

The finite-difference implementation of (6) is similar to (5). We let $z_{n v}=n v \cdot \Delta z,(n v=0, \ldots, N v)$ be the grid point in $z$ direction and $x_{m}=m \cdot \Delta x,(m=0, \ldots, M, \ldots)$ be the successive integration ranges. The difference equation of (6) can be written as follows:

$$
\begin{aligned}
& \alpha_{v, m} u_{v, n v+1}^{m}+\beta_{v, m} u_{v, n v}^{m}+\alpha_{v, m} u_{v, n v-1}^{m} \\
& =\alpha_{v, m-1} u_{v, n v+1}^{m-1}-\beta_{v, m-1} u_{v, n v}^{m-1}-\alpha_{v, m-1} u_{v, n v-1}^{m-1},
\end{aligned}
$$


where we have used the following notations:

$$
\begin{aligned}
\alpha_{v, m}= & \frac{1+j k_{0} \Delta x}{4 k_{0}^{2}(\Delta z)^{2}}, \\
\alpha_{v, m-1}= & \frac{1-j k_{0} \Delta x}{4 k_{0}^{2}(\Delta z)^{2}}, \\
\beta_{v, m}= & 1+\frac{1}{4}\left(n^{2}\left(\varepsilon_{m}, z_{n v}\right)-1\right)\left(1+j k_{0} \Delta x\right) \\
& -\frac{1+j k_{0} \Delta x}{2 k_{0}^{2}(\Delta z)^{2}}, \\
\beta_{v, m-1}= & 1+\frac{1}{4}\left(n^{2}\left(\varepsilon_{m}, z_{n v}\right)-1\right)\left(1+j k_{0} \Delta x\right) \\
& -\frac{1-j k_{0} \Delta x}{2 k_{0}^{2}(\Delta z)^{2}} .
\end{aligned}
$$

Equation (16) has expressed values at range $x_{m}$ as a function of values at range $x_{m-1}$ in the form of a linear system. When $m=1$, the values of $u_{v, n v}^{0}$ can be obtained by the same way as $u_{h, n h}^{0}$.

Equations (12) and (16) are the difference equations of the WA-3DPE decomposition model. In order to complete the equation system, we need to include equations at boundaries. For the WA-3DPE decomposition model, it contains 4 boundaries in $\pm y$ and $\pm z$ directions, respectively. Here we employ the 2nd-order accurate discretization of the derivatives $\partial u_{h} / \partial y$ and $\partial u_{v} / \partial z$. Thus, at the boundaries,

$$
\begin{aligned}
& \frac{\partial u_{h}\left(m \Delta x, y_{\max }\right)}{\partial y} \approx \frac{3 u_{h, 0}^{m}-4 u_{h, 1}^{m}+u_{h, 2}^{m}}{2 \Delta y}, \\
& \frac{\partial u_{h}\left(m \Delta x, y_{\min }\right)}{\partial y} \approx-\frac{3 u_{h, N h}^{m}-4 u_{h, N h-1}^{m}+u_{h, N h-2}^{m}}{2 \Delta y} .
\end{aligned}
$$

Equations (18) give the 2nd-order accurate finite-difference approximation of the boundary conditions at $\pm y$ :

$$
\begin{aligned}
\frac{\partial u_{v}\left(m \Delta x, z_{\max }\right)}{\partial z} & \approx \frac{3 u_{v, 0}^{m}-4 u_{v, 1}^{m}+u_{v, 2}^{m}}{2 \Delta z}, \\
\frac{\partial u_{v}(m \Delta x, 0)}{\partial z} & \approx-\frac{3 u_{v, N v}^{m}-4 u_{v, N v-1}^{m}+u_{v, N v-2}^{m}}{2 \Delta z} .
\end{aligned}
$$

Equations (19) give the 2nd-order accurate finite-difference approximation of the boundary conditions at $\pm z$.

\section{The Improved Diffraction Nonlocal BC}

4.1. Diffraction Nonlocal BC. In order to use one equation to express the diffraction nonlocal BCs of $(x, y)$ plane and $(x, z)$ plane uniformly, we use " $s$ " to represent " $y$ " and " $z$ "; therefore, for $(x, y)$ plane, $s=y$, and, for $(x, z)$ plane, $s=z$. According to $[14,15]$, the diffraction nonlocal BC in $(x, s)$ plane can be written as

$$
\frac{\partial f(x, s)}{\partial s}=-j \chi^{2} k_{0} \int_{0}^{x} \omega(x-\xi) \frac{\partial f(\xi, s)}{\partial \xi} d \xi,
$$

where the convolution kernel $\omega$ is given by

$$
\omega(x)=J_{0}\left(k_{0} x\right) e^{-j k_{0} x},
$$

where $\chi=1$ for $y=y_{\max }$ and $z=z_{\max }, \chi=-1$ for $y=y_{\min }$ and $z=0 . J_{0}$ is the zero-order Bessel function.

4.2. Recursive Convolution. According to [14], if the function in the convolution term in (20) can be expressed as $A_{i} \exp \left(B_{i}\right.$. $x$ ) then

$$
\begin{aligned}
R(x) & =\int_{0}^{x} \omega(x-\xi) \frac{\partial f(\xi, s)}{\partial \xi} d \xi=R_{i}(x) \\
& =\int_{0}^{x} A_{i} \exp \left(B_{i}(x-\xi)\right) \frac{\partial f(\xi, s)}{\partial \xi} d \xi \\
& =R_{i}(m \cdot \Delta x) \\
& =\int_{0}^{m \cdot \Delta x} A_{i} \exp \left(B_{i}(m \cdot \Delta x-\xi)\right) \frac{\partial f(\xi, s)}{\partial \xi} d \xi \\
& =R_{i}^{m} .
\end{aligned}
$$

Therefore

$$
\begin{gathered}
R_{i}^{m-1}=R_{i}[(m-1) \cdot \Delta x]=\int_{0}^{(m-1) \cdot \Delta x} A_{i} \\
\cdot \exp \left(B_{i}((m-1) \cdot \Delta x-\xi)\right) \frac{\partial f(\xi, s)}{\partial \xi} d \xi .
\end{gathered}
$$

From (22) and (23), the following recursive formula can be derived:

$$
\begin{aligned}
& R_{i}^{m} \\
& \quad=\exp (B i \cdot \Delta x) R_{i}^{m-1} \\
& \quad+\int_{(m-1) \cdot \Delta x}^{m \cdot \Delta x} A_{i} \exp \left(B_{i}(m \cdot \Delta x-\xi)\right) \frac{\partial f(\xi, s)}{\partial \xi} d \xi .
\end{aligned}
$$

In general, $\omega(x)$ is approximately expressed as

$$
\omega(x) \approx \widetilde{\omega}(x)=\sum_{i=1}^{T} A_{i} e^{B_{i} \cdot x}
$$

thus,

$$
\begin{aligned}
& R(x)=R^{m} \approx \sum_{i=1}^{T} R_{i}(x)=\sum_{i=1}^{T} R_{i}^{m} \\
& =\sum_{i=1}^{T} e^{B_{i} \cdot \Delta x} R_{i}^{m-1} \\
& \quad+\sum_{i=1}^{T} A_{i} e^{B_{i} \cdot m \Delta x} \int_{(m-1) \cdot \Delta x}^{m \cdot \Delta x} \exp \left(-B_{i} \cdot \xi \frac{\partial f(\xi, s)}{\partial \xi}\right) d \xi,
\end{aligned}
$$

where $R_{i}^{0}=0$. 
The integral term in (26) can be discretized as follows:

$$
\begin{aligned}
& \int_{(m-1) \cdot \Delta x}^{m \cdot \Delta x} \exp \left(-B_{i} \cdot \xi \frac{\partial f(\xi, v)}{\partial \xi}\right) d \xi \\
& \quad \approx \int_{(m-1) \cdot \Delta x}^{m \cdot \Delta x} \exp \left(-B_{i}\right. \\
& \left.\cdot \xi \frac{f(m \cdot \Delta x, v)-f((m-1) \cdot \Delta x, v)}{\Delta x}\right) d \xi \\
& \quad=\frac{f(m \cdot \Delta x, v)-f((m-1) \cdot \Delta x, v)}{\Delta x} \\
& \quad \times \int_{(m-1) \cdot \Delta x}^{m \cdot \Delta x} \exp \left(-B_{i} \cdot \xi\right) d \xi=\exp \left(-B_{i} \cdot m \Delta x\right) \\
& \quad \cdot\left(\frac{f(m \cdot \Delta x, v)-f((m-1) \cdot \Delta x, v)}{\Delta x}\right) \\
& \quad \times\left(\frac{1-\exp \left(-B_{i} \cdot \Delta x\right)}{-B_{i}}\right) .
\end{aligned}
$$

From (26) and (27), it follows that

$$
\begin{gathered}
j \chi^{2} k_{0} \int_{0}^{x} \omega(x-\xi) \frac{\partial f(\xi, s)}{\partial \xi} d \xi \\
=\tau \chi f^{m}-\tau \chi f^{m-1}+\chi \Psi_{m}^{f},
\end{gathered}
$$

where we have used the following notations:

$$
\begin{aligned}
\tau & =2 j k_{0} \sum_{i=1}^{T} A_{i}\left(\frac{1-e^{-B_{i} \cdot \Delta x}}{-B_{i} \cdot \Delta x}\right), \\
\Psi_{m}^{f} & =2 j k_{0} \sum_{i=1}^{T} e^{B_{i} \cdot \Delta x} R_{i}^{m-1} .
\end{aligned}
$$

In (28), the integral term in (20) is expressed by recursive convolution method.

4.3. The VF Approximation. As indicated in (25), the function $\omega(x)$ must be curve fitted with a function $\widetilde{\omega}(x)$ which is a summation of exponential terms. In $A_{i} \exp \left(B_{i} \cdot x\right), A_{i}$ and $B_{i}$ are complex numbers. To achieve this we use first the VF method [14] to express the function $g(r)=J_{0}(r)$ as a sum of exponential terms. In the VF method, the Laplace transform of $g(r)$, that is, $G(s)$, is approximated with a sum of fractions $a_{i} /\left(s-b_{i}\right)$ :

$$
G(s)=\frac{1}{\sqrt{s^{2}+1}} \approx \sum_{i=1}^{T} \frac{c_{i}}{s-d_{i}}
$$

thus

$$
g(r)=J_{0}(r) \approx \widetilde{g}(r)=\sum_{i=1}^{T} c_{i} \cdot e^{d_{i} \cdot r} .
$$

Therefore, (21) can be written as

$$
\omega(x)=J_{0}\left(k_{0} x\right) e^{-j k_{0} x} \approx \sum_{i=1}^{T} c_{i} \cdot e^{d_{i} \cdot k_{0} x} e^{-j k_{0} x} .
$$

From (25) and (32), it follows that $A_{i}=c_{i}, B_{i}=k_{0}\left(d_{i}-j\right)$. According to [14], $g(r)$ is curve fitted over the argument region $0 \leq r \leq 65000$. It was found that $T=20$ was sufficient for the computations. The values of $c_{i}$ and $d_{i}$ can be found in [14].

\section{The WA-3DPE Decomposition Model with the Improved Diffraction Nonlocal BCs}

Through formulae (18), (20), and (28), where $s=y$ and $f=$ $u_{h}$, we can obtain the following expression:

$$
u_{h, A}^{m}=\rho_{h} u_{h, B}^{m}+\eta_{h} u_{h, C}^{m}+\Xi_{h, A}^{m},
$$

where we have used the following notations:

$$
\begin{aligned}
\rho_{h} & =\frac{4}{(3+2 \tau \Delta y)}, \\
\eta_{h} & =-\frac{1}{(3+2 \tau \Delta y)}, \\
\Xi_{h, A}^{m} & =\frac{\rho_{h} \tau \Delta y}{2}\left(u_{h, A}^{m-1}\right)-\frac{\rho_{h} \Delta y}{2}\left(\Psi_{m, A}\right),
\end{aligned}
$$

where $A=0, B=1, C=2$ for $y=y_{\max }, A=N h, B=$ Nh $-1, C=N h-2$ for $y=y_{\min }$

Through formulae (19), (20), and (28) where $s=z$ and $f=u_{v}$, we can also obtain the following expression:

$$
u_{v, A}^{m}=\rho_{v} u_{v, B}^{m}+\eta_{v} u_{v, C}^{m}+\Xi_{v, A}^{m},
$$

where we have used the following notations:

$$
\begin{aligned}
\rho_{v} & =\frac{4}{(3+2 \tau \Delta z)}, \\
\eta_{v} & =-\frac{1}{(3+2 \tau \Delta z)}, \\
\Xi_{v, A}^{m} & =\frac{\rho v \tau \Delta z}{2}\left(u_{v, A}^{m-1}\right)-\frac{\rho_{v} \Delta z}{2}\left(\Psi_{m, A}\right),
\end{aligned}
$$

where $A=0, B=1, C=2$ for $z=z_{\max }, A=N v, B=$ $N v-1, C=N v-2$ for $z=0$.

Equations (33) and (35) are the two-dimensional improved diffraction nonlocal BCs in $(x, y)$ plane and $(x, z)$ plane, respectively. As the improved diffraction nonlocal BCs are linear equations, they can be applied in (12) and (16) directly. From (12) and the BC (33), the following global matrix equation is obtained:

$$
\Lambda_{h, m} U_{h, m}=\Lambda_{h, m-1} U_{h, m-1}+\Omega_{h} .
$$

From (16) and the BC (35), the following global matrix equation is obtained:

$$
\Lambda_{v, m} U_{v, m}=\Lambda_{v, m-1} U_{v, m-1}+\Omega_{v}
$$

Equations (37) and (38) are the WA-3DPE decomposition model with the improved diffraction nonlocal BCs. The details of the matrices/vectors are given in Appendix. 


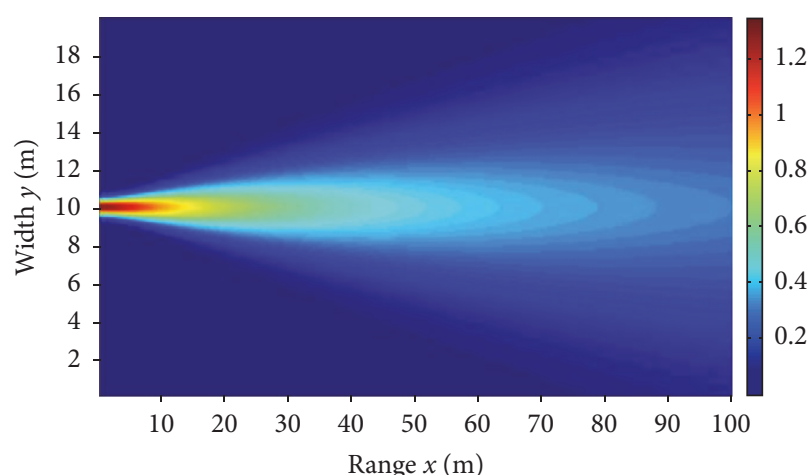

(a)

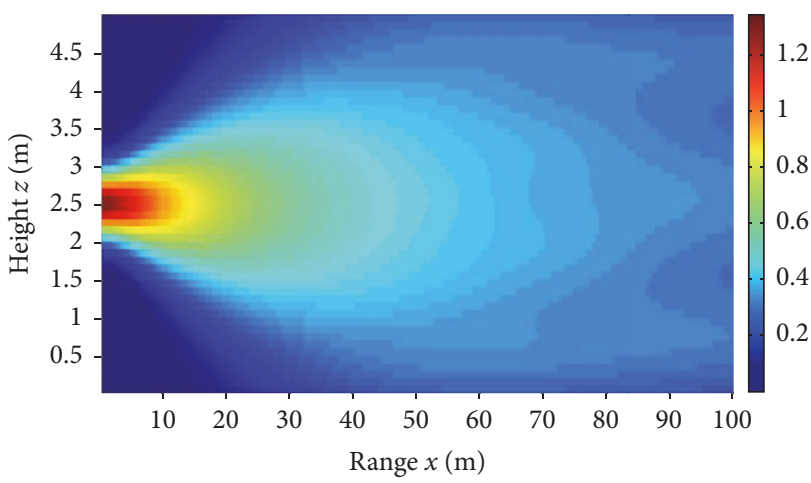

(c)

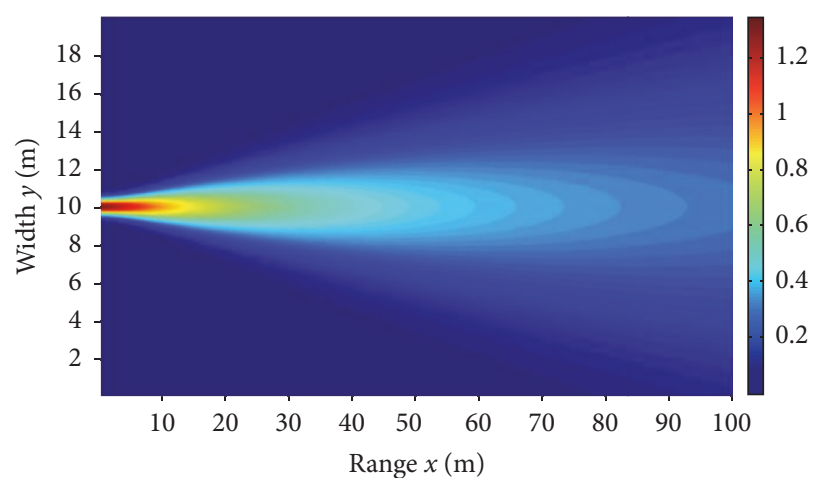

(b)

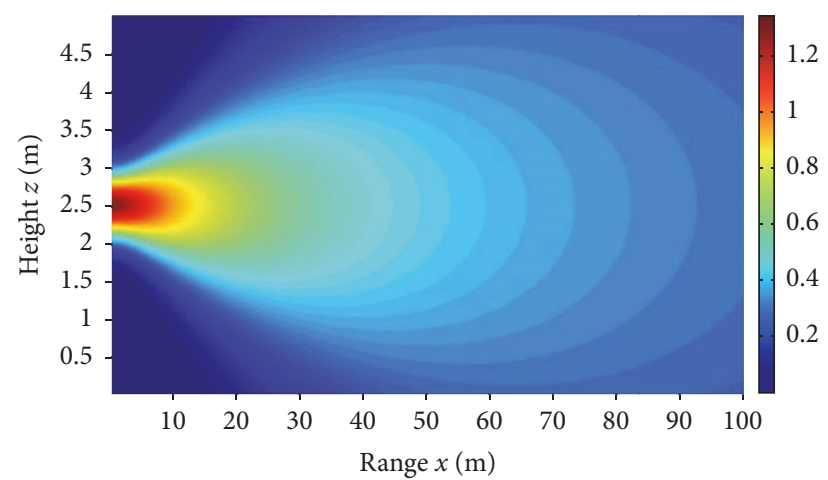

(d)

FIGURE 1: Pseudocolor maps of field amplitude. Field amplitude of $u_{h}$ in $(x, y)$ plane and at $z=2.5 \mathrm{~m}$, computed by (a) the standard 3DPE decomposition model with the window function BCs; (b) the WA-3DPE decomposition model with the improved diffraction nonlocal BCs. Field amplitude of $u_{v}$ in $(x, z)$ plane and at $y=10 \mathrm{~m}$, computed by (c) the standard 3DPE decomposition model with the window function BCs; (d) the WA-3DPE decomposition model with the improved diffraction nonlocal BCs.

\section{Numerical and Experimental Results}

6.1. Free Space Propagation. The biggest advantage of diffraction nonlocal BCs is that it can be used in conjunction with any initial source. In the following computation, we choose Gaussian source [15] as the initial field. We consider radio wave propagation along the positive direction of $x$-axis in free space, which means that the improved diffraction nonlocal $\mathrm{BC}$ can be applied in $\pm y$ and $\pm z$ four boundaries. The sizes of computational region are that $z_{\max }=5 \mathrm{~m}, z_{\min }=0 \mathrm{~m}$, $y_{\max }=20 \mathrm{~m}, y_{\min }=0 \mathrm{~m}, x_{\max }=100 \mathrm{~m}$, and $x_{\min }=$ $0 \mathrm{~m}$. The transmitting antenna is fixed at the point $(x=$ $0, y=10, z=2.5)$. The transmitting antenna frequency is $3 \mathrm{GHz}$; the $3 \mathrm{~dB}$ beamwidth is $5 \mathrm{deg}$ and elevation angle is $0 \mathrm{deg}$. The refractive index $n=1$. In order to verify the computational accuracy and efficiency of the WA-3DPE decomposition model with the improved diffraction nonlocal $\mathrm{BCs}$, we choose the computation results of the standard 3DPE decomposition model [16] as a comparison. As it is accurate when the elevation angle is $0 \mathrm{deg}$, the standard 3DPE decomposition model computation results can be chosen as a comparison. The standard 3DPE decomposition model was solved by the Split-Step Fourier Transform (SSFT) method with the window function BCs. Figures 1(a) and 1(b) are the pseudocolor map of field amplitude of $u_{h}$ in $(x, y)$ plane and at $z=2.5 \mathrm{~m}$. Figure $1(\mathrm{a})$ gives the standard 3DPE decomposition model with window function BC computation results and Figure 1(b) shows the WA3DPE decomposition model with the improved diffraction nonlocal BC computation results. Figures $1(\mathrm{c})$ and $1(\mathrm{~d})$ are the pseudocolor map of field amplitude of $u_{v}$ in $(x, z)$ plane and at $y=10 \mathrm{~m}$. Figure 1(c) gives the standard 3DPE decomposition model with window function $\mathrm{BC}$ computation results and Figure 1(d) shows the WA-3DPE decomposition model with the improved diffraction nonlocal $\mathrm{BC}$ computation results.

Table 1 gives some specific parameters involved in the computation of the WA-3DPE decomposition model and the standard 3DPE decomposition model. The computer CPU is the Intel(R) Core(TM) i5-2400@3.1 GHz. For the standard 3DPE decomposition model solved by SSFT, four window functions are necessary in $\pm y$ and $\pm z$ directions. The window function in the computation is Hanning window function [8]. Theoretically, the greater the thickness of the absorbing layer is, the better the absorbing effect is; but for large-scale radio propagation problems in complex environments, the thickness of the absorbing layer cannot be large enough due to the restriction of computation amount. In our computation, the thickness of the absorbing layer is 20 times larger than the computation area in $y$ and $z$ directions. The size of the actual computation area of the standard 3DPE decomposition 


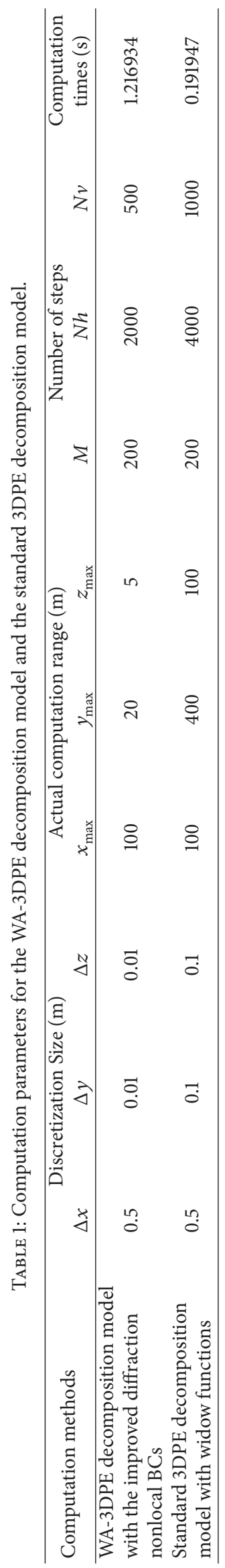


TABLE 2: Parameters of knife edge propagation.

\begin{tabular}{lc}
\hline Names & Values \\
\hline Tx antenna height & $0.8 \mathrm{~cm}$ \\
Rx antenna height & $1 \mathrm{~cm}$ \\
Range & $2.5 \mathrm{~m}$ \\
Width & $1 \mathrm{~m}$ \\
$d 1$ & $30 \mathrm{~cm}$ \\
$d 2$ & $25 \mathrm{~cm}$ \\
$w 1$ & $84.7 \mathrm{~cm}$ \\
$w 2$ & $89 \mathrm{~cm}$ \\
$h 1$ & $6.5 \mathrm{~cm}$ \\
$h 2$ & $4.9 \mathrm{~cm}$ \\
\hline
\end{tabular}

model in $y$ direction reaches $y_{\max }=400 \mathrm{~m}$. It means that the total thickness of the absorbing layer is $380 \mathrm{~m}$ and $190 \mathrm{~m}$ in the $+y$ and $-y$ direction, respectively. The size of the actual computation area of the standard 3DPE decomposition model in $z$ direction reaches $y_{\max }=100 \mathrm{~m}$. It means that the total thickness of the absorbing layer is $95 \mathrm{~m}$ and $47.5 \mathrm{~m}$ in the $+z$ and $-z$ direction, respectively. It can be seen from Table 1 that the computational speed of the standard 3DPE decomposition model is faster than the WA-3DPE decomposition model. But it is easy to find in Figure 1 that the computational accuracy of the WA-3DPE decomposition model is higher than the standard 3DPE decomposition model. Besides, it can be found that although the discretization size of FD is more restrictive than SSFT, the improved nonlocal BCs can absorb wave completely without increasing extra computation space. For the window function $\mathrm{BCs}$, it is difficult to absorb the wave completely even if the computation space is further increased.

Paper [14] had already investigated the improvement of computation speed brought by RC formulation with VF method, so it does not need to be analyzed again. For the above-mentioned 3D problem, the computing time of Dalrymple and Martin method [14] is 18.9054 s.

6.2. Knife Edge Propagation. To verify the effectiveness of the WA-3DPE decomposition model with the improved diffraction nonlocal BCs, knife edge propagation problems are computed and measured. As depicted in Figure 2, two knife edges are center symmetrically situated on a metal plane. The parameters of their size and location are given in Table 2.

The measurements are made by transmitting a continuous wave signal which are generated by the Agilent Signal Generator. The transmitter output power is $0 \mathrm{dBm}$. The transmitting antenna for the measurements is a quarter wavelength monopole antenna, and the gain of it is ignored. The Agilent Spectrum Analyzer is used as a receiver with very narrow bandwidth. The receiving antenna is a waveguide antenna, having a gain of $0 \mathrm{dBi}$ and working at the frequencies from $8 \mathrm{GHz}$ to $10 \mathrm{GHz}$. According to the aforementioned properties of experiment equipment, the working frequency is chosen at $9.375 \mathrm{GHz}$. Both the transmitting antenna and

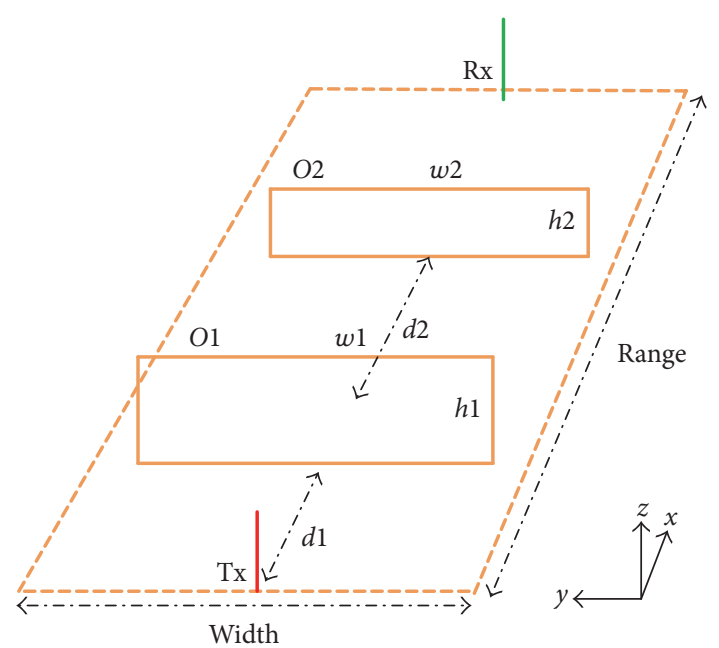

Figure 2: Knife edge propagation.

receiving antenna are set on an aluminous plane which is surrounded by wave-absorbing materials to simulate free space propagation environment.

Both of the single knife edge propagation and double knife edges propagation are simulated and measured. In the simulation and experiment of single knife edge propagation, we only keep the knife edge $\mathrm{O} 1$ and take the knife edge $\mathrm{O} 2$ away. The curves of propagation loss varying with range are given in Figure 3. Figure 3(a) gives the simulation and experiment results of the single knife edge propagation. Then we put on the knife edge $\mathrm{O} 2$ and measured again. Figure 3(b) gives the simulation and experiment results of the double knife edges propagation. In order to increase the comparability, we also give the MoM (Method of Moment) and WA-2DPE (wide-angle two-dimensional parabolic equation) computation results in Figure 3. In the simulations, the surface BC is set as PEC, which means the improved diffraction nonlocal $\mathrm{BC}$ is applied in up, left, and right boundaries. The results of MoM are obtained by the commercial solver FEKO 7.0.

It can be seen from Figure 3 that the results of the WA3DPE decomposition model with the improved diffraction nonlocal BCs, MoM, measurement, and WA-2PDE are basically the same no matter for the single knife edge propagation or the double knife edge propagation. However, there exists a big difference in which MoM computation presents fluctuant results while the WA-3DPE decomposition model with the improved diffraction nonlocal BCs and measurement does not. This is because PE only deals with the forward propagation wave, and the signal fluctuation caused by forward propagation and backward propagation interference is not modeled in front of the edges. Theoretically, the measurement should also present fluctuant results in front of the edges, but they are not be shown in Figure 3 in that the receiving antenna in our experiment is a waveguide antenna, which can only receive forward propagation waves. The computation results of WA-3DPE are more closer to measurement results than those of WA-2PDE, as the WA-3DPE considers the horizontal diffraction propagation. 


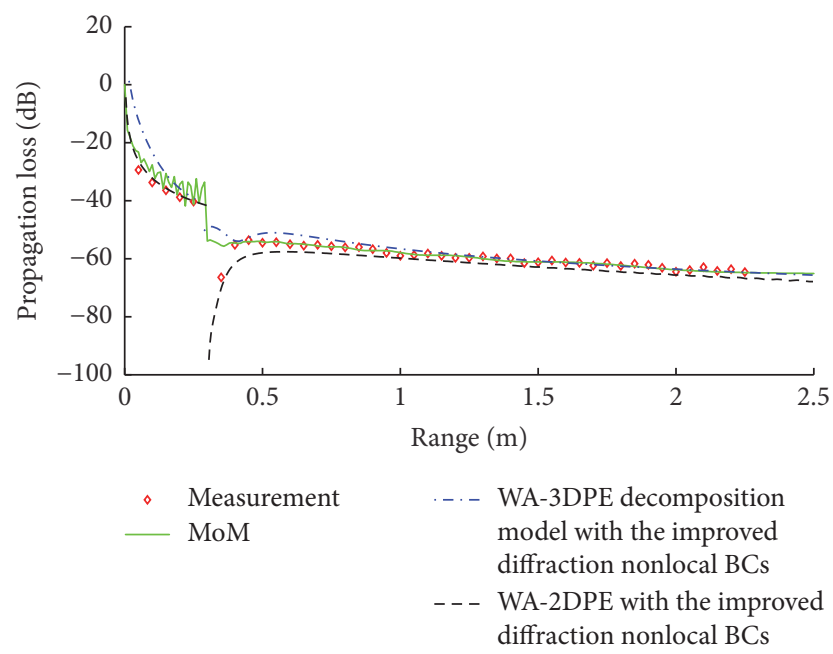

(a)

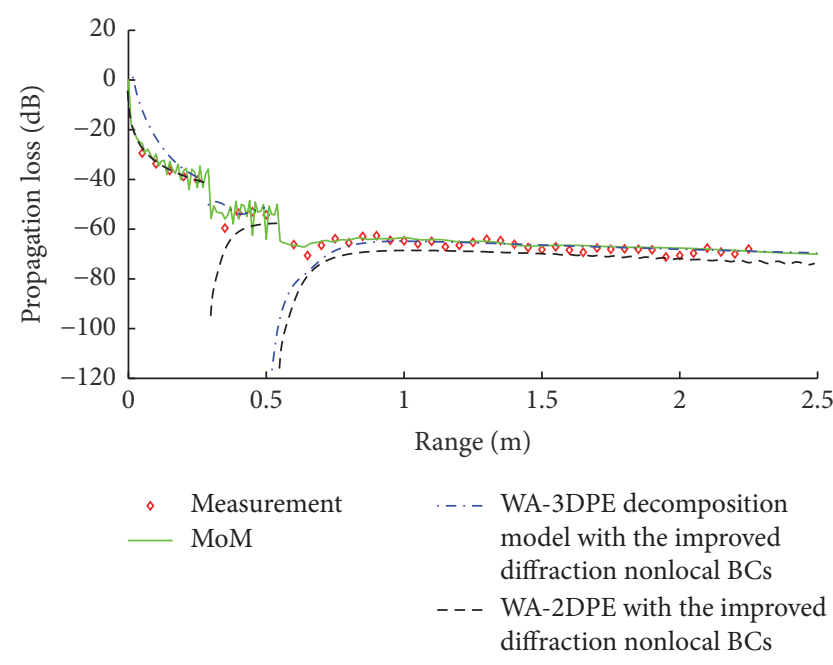

(b)

FIGURE 3: Comparison of the results of the WA-3DPE decomposition model with the improved diffraction nonlocal BCs with the measurement, MoM, and WA-2PDE results: (a) propagation loss versus range for single knife edge and (b) propagation loss versus range for double knife edges.

\section{Conclusion}

In order to accelerate the computational speed, we proposed to apply the RC formulation with VF method in the diffraction nonlocal BC. Furthermore, the improved diffraction nonlocal BC with RCs was applied in the WA-3DPE decomposition model. The computational accuracy and speed of the WA-3DPE decomposition model with diffraction nonlocal $\mathrm{BC}$ with RC formulation were demonstrated through the comparison with the standard 3DPE decomposition model, $\mathrm{MoM}$, measurement, and WA-2PDE. The proposed WA3DPE decomposition model with the improved diffraction nonlocal $\mathrm{BC}$ with $\mathrm{RC}$ formulation is suitable for the predictions of radio wave propagation in small areas like urban district environments.

\section{Appendix}

$$
\begin{aligned}
& \Lambda_{h, m}=\left[\begin{array}{cccccc}
\beta_{h, m}^{\prime} & \alpha_{h, m}^{\prime} & 0 & \cdots & 0 & 0 \\
\alpha_{h, m} & \beta_{h, m} & \alpha_{h, m} & \cdots & 0 & 0 \\
0 & \alpha_{h, m} & \beta_{h, m} & \alpha_{h, m} & \cdots & 0 \\
\vdots & \vdots & \vdots & \vdots & \vdots & \vdots \\
0 & \cdots & \alpha_{h, m} & \beta_{h, m} & \alpha_{h, m} & 0 \\
0 & 0 & \cdots & \alpha_{h, m} & \beta_{h, m} & \alpha_{h, m} \\
0 & 0 & 0 & \cdots & \alpha_{h, m}^{\prime} & \beta_{h, m}^{\prime}
\end{array}\right], \\
& \beta_{h, m}^{\prime}=\beta_{h, m}+\rho_{h} \alpha_{h, m},
\end{aligned}
$$

$\alpha_{h, m}^{\prime}=\alpha_{h, m}+\eta_{h} \alpha_{h, m}$

$\Lambda_{h, m-1}$

$=\left[\begin{array}{cccccc}\beta_{h, m-1} & \alpha_{h, m-1} & 0 & \cdots & 0 & 0 \\ \alpha_{h, m-1} & \beta_{h, m-1} & \alpha_{h, m-1} & \cdots & 0 & 0 \\ 0 & \alpha_{h, m-1} & \beta_{h, m-1} & \alpha_{h, m-1} & \cdots & 0 \\ \vdots & \vdots & \vdots & \vdots & \vdots & \vdots \\ 0 & \cdots & \alpha_{h, m-1} & \beta_{h, m-1} & \alpha_{h, m-1} & 0 \\ 0 & 0 & \cdots & \alpha_{h, m-1} & \beta_{h, m-1} & \alpha_{h, m-1} \\ 0 & 0 & 0 & \cdots & \alpha_{h, m-1} & \beta_{h, m-1}\end{array}\right]$,

$\Omega_{h}=\left[\begin{array}{c}-u_{h, 0}^{m-1} \\ \vdots \\ -u_{h, N h}^{m-1}\end{array}\right]-\left[\begin{array}{c}\Xi_{h, 0}^{m} \\ \vdots \\ \Xi_{h, N h}^{m}\end{array}\right]$,

$U_{h, m}=\left[\begin{array}{c}u_{h, 1}^{m} \\ \vdots \\ u_{h, N h-1}^{m}\end{array}\right]$,

$U_{h, m-1}=\left[\begin{array}{c}u_{h, 1}^{m-1} \\ \vdots \\ u_{h, N h-1}^{m-1}\end{array}\right]$, 


$$
\begin{aligned}
& \Lambda_{v, m}=\left[\begin{array}{cccccc}
\beta_{v, m}^{\prime} & \alpha_{v, m}^{\prime} & 0 & \cdots & 0 & 0 \\
\alpha_{v, m} & \beta_{v, m} & \alpha_{v, m} & \cdots & 0 & 0 \\
0 & \alpha_{v, m} & \beta_{v, m} & \alpha_{v, m} & \cdots & 0 \\
\vdots & \vdots & \vdots & \vdots & \vdots & \vdots \\
0 & \cdots & \alpha_{v, m} & \beta_{v, m} & \alpha_{v, m} & 0 \\
0 & 0 & \cdots & \alpha_{v, m} & \beta_{v, m} & \alpha_{v, m} \\
0 & 0 & 0 & \cdots & \alpha_{v, m}^{\prime} & \beta_{v, m}^{\prime}
\end{array}\right], \\
& \beta_{v, m}^{\prime}=\beta_{v, m}+\rho_{v} \alpha_{v, m}, \\
& \alpha_{v, m}^{\prime}=\alpha_{v, m}+\eta_{v} \alpha_{v, m} \text {, } \\
& \Lambda_{v, m-1} \\
& =\left[\begin{array}{cccccc}
\beta_{v, m-1} & \alpha_{v, m-1} & 0 & \cdots & 0 & 0 \\
\alpha_{v, m-1} & \beta_{v, m-1} & \alpha_{v, m-1} & \cdots & 0 & 0 \\
0 & \alpha_{v, m-1} & \beta_{v, m-1} & \alpha_{v, m-1} & \cdots & 0 \\
\vdots & \vdots & \vdots & \vdots & \vdots & \vdots \\
0 & \cdots & \alpha_{v, m-1} & \beta_{v, m-1} & \alpha_{v, m-1} & 0 \\
0 & 0 & \cdots & \alpha_{v, m-1} & \beta_{v, m-1} & \alpha_{v, m-1} \\
0 & 0 & 0 & \cdots & \alpha_{v, m-1} & \beta_{v, m-1}
\end{array}\right], \\
& \Omega_{v}=\left[\begin{array}{c}
-u_{v, 0}^{m-1} \\
\vdots \\
-u_{v, N v}^{m-1}
\end{array}\right]-\left[\begin{array}{c}
\Xi_{v, 0}^{m} \\
\vdots \\
\Xi_{v, N v}^{m}
\end{array}\right] \text {, } \\
& U_{v, m}=\left[\begin{array}{c}
u_{v, 1}^{m} \\
\vdots \\
u_{v, N v-1}^{m}
\end{array}\right] \text {, } \\
& U_{v, m-1}=\left[\begin{array}{c}
u_{v, 1}^{m-1} \\
\vdots \\
u_{v, N v-1}^{m-1}
\end{array}\right] \text {. }
\end{aligned}
$$

\section{Conflicts of Interest}

The authors declare that there are no conflicts of interest regarding the publication of this paper.

\section{Acknowledgments}

This work was supported by National Key Technology Support Program 2015BAK05B01: research on a new generation news-gathering system based on ultrahigh speed WLAN and BD-2.

\section{References}

[1] M. F. Levy, "Parabolic equation modelling of propagation over irregular terrain," Electronics Letters, vol. 26, no. 15, pp. 1153$1155,1990$.
[2] L. Saini and U. Casiragh, "A 3D Fourier split-step technique for modelling microwave propagation in urban areas," in Proceedings of European Conference on Radio Relay Systems IET, pp. 210-214, 2002.

[3] C. A. Zelley and C. C. Constantinou, "A three-dimensional parabolic equation applied to VHF/UHF propagation over irregular terrain," IEEE Transactions on Antennas and Propagation, vol. 47, no. 10, pp. 1586-1596, 1999.

[4] M. F. Levy, "Diffraction studies in urban environment with wide-angle parabolic equation method," Electronics Letters, vol. 28, no. 16, pp. 1491-1492, 1992.

[5] A. A. Zaporozhets and M. F. Levy, "Modelling of radiowave propagation in urban environment with parabolic equation method," Electronics Letters, vol. 32, no. 17, pp. 1615-1616, 1996.

[6] R. Janaswamy and J. B. Andersen, "Path loss predictions in urban areas sprawling over irregular terrain," in Proceedings of the 1998 9th IEEE International Symposium on Personal, Indoor and Mobile Radio Communications, PIMRC. Part 3 (of 3), pp. 874-878, September 1998.

[7] J. R. Kuttler and G. D. Dockery, "Theoretical description of the parabolic approximation/Fourier split-step method of representing electromagnetic propagation in the troposphere," Radio Science, vol. 26, no. 2, pp. 381-393, 1991.

[8] P. Zhang, L. Bai, Z. Wu, and F. Li, "Effect of window function on absorbing layers top boundary in parabolic equation," in Proceedings of the 3rd Asia-Pacific Conference on Antennas and Propagation, APCAP 2014, pp. 849-852, chn, July 2014.

[9] J.-P. Berenger, "A perfectly matched layer for the absorption of electromagnetic waves," Journal of Computational Physics, vol. 114, no. 2, pp. 185-200, 1994.

[10] M. Ehrhardt and A. Zisowsky, "Discrete non-local boundary conditions for split-step Padé approximations of the one-way Helmholtz equation," Journal of Computational and Applied Mathematics, vol. 200, no. 2, pp. 471-490, 2007.

[11] G. H. Song, "Transparent boundary conditions for beampropagation analysis from the green's function method," Journal of the Optical Society of America A: Optics and Image Science, and Vision, vol. 10, no. 5, pp. 896-904, 1993.

[12] P. Zhang, L. Bai, Z. Wu, and L. Guo, "Applying the Parabolic Equation to Tropospheric Groundwave Propagation: A review of recent achievements and significant milestones.," IEEE Antennas and Propagation Magazine, vol. 58, no. 3, pp. 31-44, 2016.

[13] Z. Ge, G. Lu, and R. Wang, "Using the finite difference parabolic equation with nonlocal boundary condition for complex environment radiowave propagation prediction," in Proceedings of the 11th International Symposium on Antennas, Propagation and EM Theory, ISAPE 2016, pp. 478-480, chn, October 2016.

[14] C. Mias, "Fast computation of the nonlocal boundary condition in finite difference parabolic equation radiowave propagation simulations," Institute of Electrical and Electronics Engineers. Transactions on Antennas and Propagation, vol. 56, no. 6, pp. 1699-1705, 2008.

[15] M. F. Levy, Parabolic Equation Methods for Electromagnetic Wave Propagation, vol. 45, The Institution of Electrical Engineers, London, UK, 1st edition, 2000.

[16] G. Lu, R. Wang, Z. Cao, and K. Jiang, "A decomposition method for computing radiowave propagation loss using threedimensional parabolic equation," Progress In Electromagnetics Research M, vol. 44, pp. 183-189, 2015. 


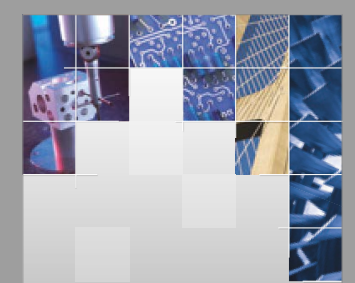

\section{Enfincering}
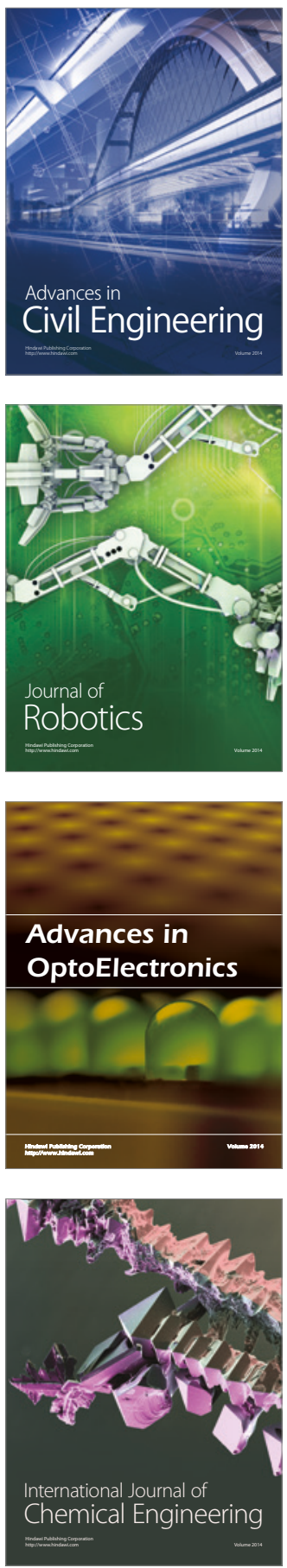

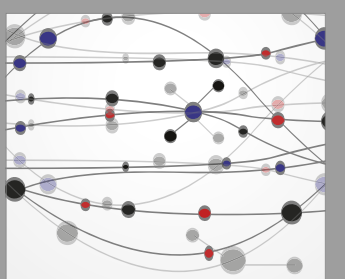

The Scientific World Journal

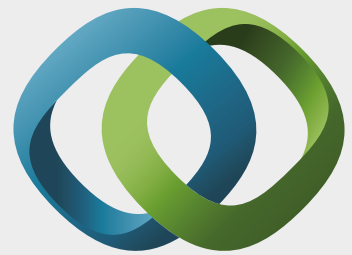

\section{Hindawi}

Submit your manuscripts at

https://www.hindawi.com
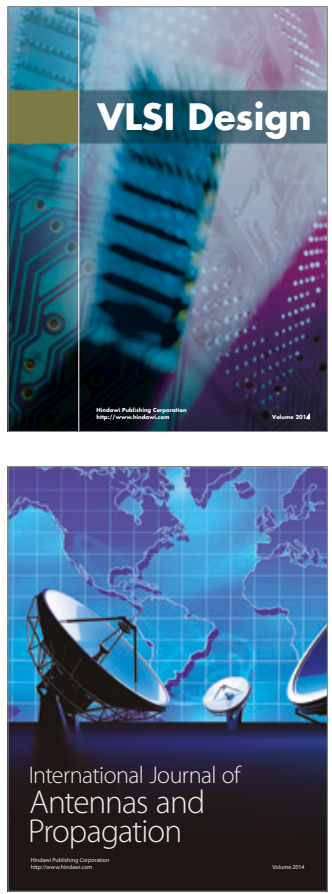

\section{Rotating}

Machinery
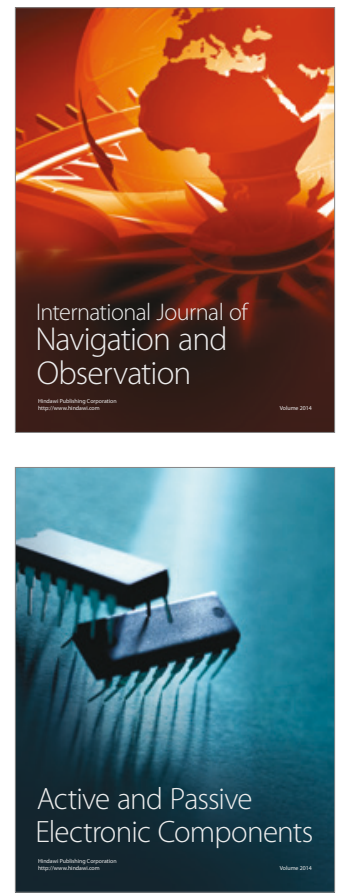
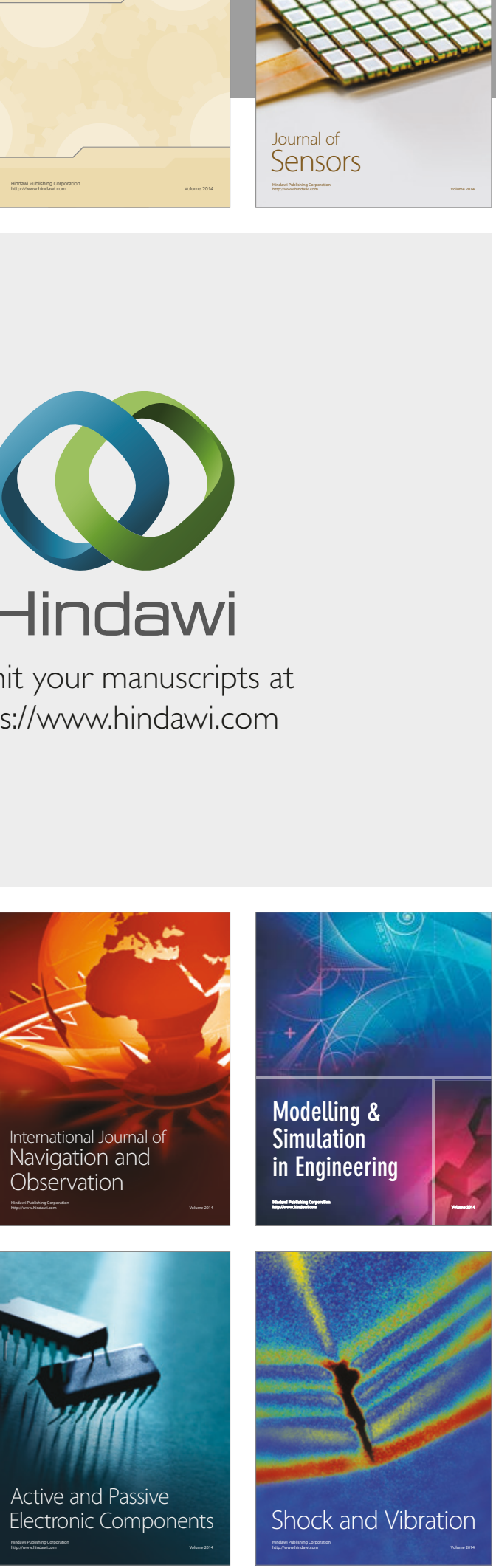
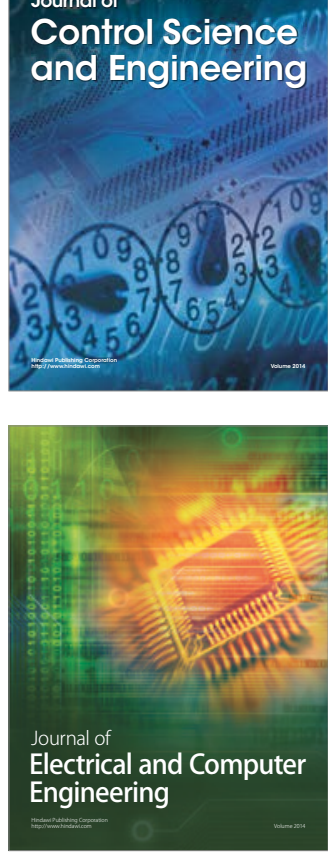

Distributed

Journal of

Control Science

and Engineering
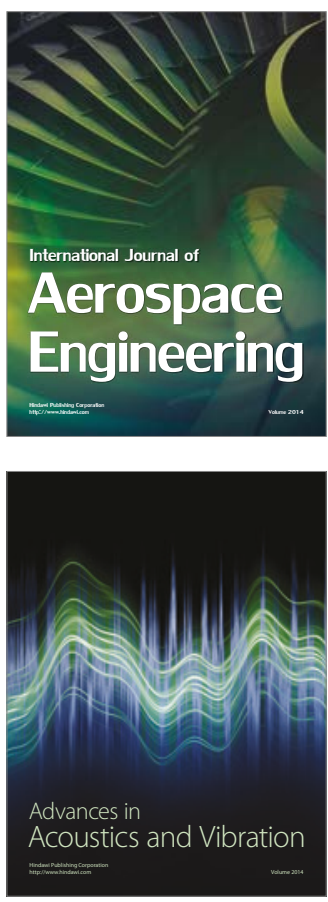

Sensor Networks 